\title{
Utilizing Data from the NOAA National Data Buoy Center
} US Army Corps of Engineers ${ }_{\circledast}$

By Candice Hall and Robert E. Jensen

PURPOSE: This Coastal and Hydraulics Engineering Technical Note (CHETN) guides users through the quality control (QC) and processing steps that are necessary when using archived U.S. National Oceanic and Atmospheric Administration (NOAA) National Data Buoy Center (NDBC) wave and meteorological data. This CHETN summarizes methodologies to geographically clean and QC NDBC measurement data for use by the U.S. Army Corps of Engineers (USACE) user community.

INTRODUCTION: The USACE acknowledges that "Estimates of wave conditions are needed in almost all coastal engineering studies" (USACE 2002, II-1-1). The expense to monitor waves along all U.S. coasts can become cost prohibitive; however, the importance of wave measurements is real. The USACE has adopted a paradigm of strategically positioning wave measurement sites to maximize their effectiveness and filling in the gaps with model estimates. To satisfy this requirement, in the 1970s, the USACE developed the Wave Information Studies (WIS) that computes long-term (over 4 decades), hourly wave estimates along all U.S. coastlines, including the Great Lakes and U.S. island territories (USACE 2020).

The WIS wave estimates require in situ buoy wave data for validation and calibration similar to that of Ortiz-Royero and Mercado-Irizarry (2008); Reguero et al. (2012); Rusu and Guedes Soares (2012); Van Nieuwkoop et al. (2013); Stopa and Cheung (2014); Stopa and Mouche (2016). One source of WIS validation data is the NDBC network of meteorological and wave measurement buoys.

NDBC has deployed buoys with wave measurement capabilities around the U.S. coastline and the Great Lakes since the 1970s. Long-term time series data from a single buoy site have experienced multiple instrumentation modifications and data archival upgrades with advances in technology. Between 1970 and 2020, NDBC has deployed at least eight directional wave measurement systems for operational or experimental use (e.g., Steele et al. 1985; NDBC 1996; NDBC 2003; Teng et al. 2007; Crout et al. 2008; Teng et al. 2009; Riley et al. 2011; Hall et al. 2018a; Riley et al. 2019).

While NDBC has worked hard to minimize the effects of the modifications on its data (e.g., Teng and Timpe 1995; Teng et al. 2007; Riley and Bouchard 2015; Hall et al. 2018a), different versions of these data are archived in multiple online locations, each with their own set of storage protocols. If these data are used without prior knowledge of these archival idiosyncrasies, use of the measured data in wave-related research activities may be compromised. This CHETN summarizes the steps required to achieve the best available time-series datasets for all buoy data collected by NDBC since the 1970s. 
NDBC ARCHIVAL DATA SOURCES: NDBC currently archives its data in three places:

1. Source 1: NDBC website individual station pages: Historical data (e.g., for Station 44014: https://www.ndbc.noaa.gov/station page.php? station=44014).

2. Source 2: The official NOAA archives, which are located at the National Centers for Environmental Information (NCEI) (e.g., https://www.ncei.noaa.gov/access/marineenvironmental-buoy-database).

3. Source 3: The NDBC Distributed Oceanographic Data Systems framework, which uses the Thematic Real-time Environmental Distributed Data Services (THREDDS) (e.g., https://dods.ndbc.noaa.gov/thredds/catalog/data/catalog.html).

The NDBC website (Source 1) stores data in compressed text files while the NCEI and THREDDS data (Sources 2 and 3, respectively) are stored in Unidata's Network Common Data Form (netCDF) files. NDBC collates their website data (Source 1) annually and mirrors these data on the NDBC THREDDS (Source 3) in netCDF format, meaning that these may be considered as a single source of NDBC data that are stored in different formats. This CHETN describes data storage protocols and issues between the first two, non-matching archival sources: the NDBC website (Source 1) and the official NOAA archives at NCEI (Source 2). For simplicity, NDBC data sourced from the NDBC website will be defined as "NDBC" data, while NDBC data sourced from the official NOAA Archives at the NCEI will defined as "NCEI" data. The reader should remember throughout this CHETN that the data should be identical as they are collected from the same buoy site and instrument. The user should also be cognizant of the availability of different variables within these datasets, which are an expected result of altered sampling protocols, instrumentation and technological advances over the decades. Of note is that the scope of this work only covers data collected by NDBC and not partner data that are sent to NDBC for publishing (e.g., buoy stations funded and maintained by universities and other agencies), as NDBC does not QC those partner data.

NDBC Website Data. These published NDBC data are the most accurate to date, as they have been subjected to manual QC by NDBC Mission Control Center (MCC) data analysts (NDBC 2009). They display the most consistent structure in reported variables and nomenclature over time. These datasets typically contain NDBC wave data: significant wave height, dominant (peak spectral) and average (mean) periods, mean wave direction at the peak frequency, spectral wave density data $\left(\mathrm{c}_{11}\right)$, the four Fourier directional parameters for spectral wave direction data (alpha alpha $2, r_{1}$, and $r_{2}$ ), and meteorological data: wind direction, wind speed, wind gust, air pressure at sea level, air temperature, and sea surface temperature. NDBC has consistently documented parameter definitions and methodologies applied in the resulting wave measurements (Steele et al. 1985; NDBC 2009; NDBC 2018a; NDBC 2018b).

Of note is that NDBC data are stored with only dates and time, and no other metadata. There are no indications of changes in buoy location or buoy systems (e.g., hull characteristics, instrumentation characteristics, nor sensor elevation above the water surface). This omission leads to multiple identification issues for end-users. For example, when deriving 10-meter winds, what were the anemometer heights, air, and water temperatures? For determining the buoy location (which is not always a given even for moored buoys as they sometimes break loose from their mooring and go adrift; or they are physically pulled aboard a ship for regular servicing; or the station location is changed), what were the Global Positioning System (GPS) metadata? 
Additionally, data stored without metadata do not indicate which instrumentation (sensor-payload) are used to collect the data. As most NDBC buoys presently contain secondary (redundant) meteorological sensors, MCC data analysts switch between sensors as necessary to ensure an unbroken data stream from each station. However, most instrumentation have their own collection and processing protocols and algorithms, and these differences manifest in the final data product.

NCEI NetCDF Data. NetCDF NDBC datasets stored at NCEI are considered by NOAA to be its official archived data source. In 2011, NDBC implemented a procedure to store data in netCDF format on the NCEI server. Since then, archived NDBC datasets have been sourced from the NDBC real-time data stream (instead of the NDBC database), which, due to latency commitments, are only subjected to very broad, automatic QC protocols*. In other words, these data are not manually reviewed for accuracy. The NDBC netCDF construction process also introduces errors into the file structure. These generated netCDF files were not reviewed for errors prior to publishing until 2018.

Prior to 2011, NCEI data were stored in coded form (required a decoder to access the data). In 2020, NDBC converted the pre-2011 data files into netCDF format. This conversion resulted in different netCDF structures and file naming conventions for the pre- and post-2011 data files. Regardless, all NCEI-sourced NDBC data are now in netCDF format.

Fortuitously, the NCEI datasets do contain metadata, such as GPS positions, primary and secondary (where available) sensor information, and software versions. Data that are collected during time periods where the buoys were adrift, or during servicing periods, are identifiable, as are the different instruments that were used to collect the published data.

While the NCEI datasets include all the NDBC meteorological and spectral wave information that are stored on the NDBC website, they also include uncorrected spectral energy wave data $\left(\mathrm{c}_{11 \mathrm{~m}}\right)$ and spectral co- and quad-spectra $\left(\mathrm{C}_{12}, \mathrm{C}_{13}, \mathrm{C}_{22}, \mathrm{C}_{33}, \mathrm{Q}_{12}, \mathrm{Q}_{13}, \mathrm{C}_{22}, \mathrm{C}_{33}, \mathrm{C}_{23}\right.$, and $\left.\mathrm{Q}_{23}\right)$ as well as four data quality assurance parameters (gamma2, gamma $, \mathrm{Phi}_{\mathrm{h}}, \mathrm{R} / \mathrm{q}$ ): see Appendix A for explanations of these parameters. These additional spectral wave data allow for the recalculation of spectral wave energy and integral wave parameters to circumvent any processing applied to these data during NDBC shoreside protocols.

PROCESS TO CREATE A BEST AVAILABLE NDBC DATASET: As the NDBC website and NCEI netCDF data files provide complementary information, both should be combined to develop a best available dataset for each NDBC station location.

NDBC Website Data Downloads. NDBC wave and meteorological data are stored in multiple files within the "Historical Data \& Climatic Summaries" page of the individual station's webpages (NDBC 2020). Data are stored in text files and zipped by month for the current year and by year for the previous years (Table 1). Individual files are stored per dataset type using the following associated file nomenclatures (in parentheses) and file codes [in square brackets]: standard meteorological (stdmet [h]); spectral wave density (swden [w]); spectral wave (alpha 1 ) direction (swdir [d]); spectral wave (alpha 2 ) direction (swdir2 [i]); spectral wave ( $\mathrm{r}_{1}$ ) direction (swr1 [j]);

* S. DiNapoli. October 2018. NDBC. Personal communication. 
and spectral wave $\left(\mathrm{r}_{2}\right)$ direction (swr2 [k]). Downloaded files require unzipping to access the data in text format.

\begin{tabular}{|c|c|}
\hline Yearly data file URL & https://www.ndbc.noaa.gov/data/historical/swden/46029w1996.txt.gz \\
\hline Monthly data file URL & https://www.ndbc.noaa.gov/data/swden/Jan/4602912020.txt.gz \\
\hline
\end{tabular}

NCEI NetCDF Data Downloads. NDBC wave and meteorological data are stored in the NCEI Coastal Buoy Data Archive with unique "Station" identification webpages (NCEI 2020a). Data are stored in netCDF files by month for each year. When a buoy is serviced, a second deployment (D) netCDF file is created for the balance of the month, meaning that multiple deployment files may be available for a single month. The deployment number designation can reach up to 10 (as of August 2020). Note that the "10" does not mean that the station was serviced 10 times in 1 month.

NCEI has modified its website and server URLs to reflect its name change (the National Oceanographic Data Center was reformed as the National Center for Environmental Research). Table 2 shows the different pre- and post-2011 URLs and naming conventions. For more information on the netCDF data file naming conventions, see the NCEI information about the netCDF data files in the NOAA Marine Environmental Buoy Database (NCEI 2020b).

\section{Table 2. NCEI download URLs for NDBC netCDF data files.}

NCEI Download URL for Station 44014 prior to April 2020

\begin{tabular}{|l|l|l|}
\hline $\begin{array}{l}\text { Pre- } \\
2011\end{array}$ & https://data.nodc.noaa.gov/thredds/fileServer/ndbc/cmanwx/2006/12/44014_200612.nc
\end{tabular}

Post-
2011 https://data.nodc.noaa.gov/thredds/fileServer/ndbc/cmanwx/2011/01/NDBC_44014_201101_D1_v00.nc

NCEI Download URL for Station 41001 after April 2020

\begin{tabular}{l|l} 
Pre- \\
2011
\end{tabular} https://www.ncei.noaa.gov/data/oceans/ndbc/cmanwx/1980/01/41001_198001.nc

Post-
2011 https://www.ncei.noaa.gov/data/oceans/ndbc/cmanwx/2020/04/NDBC_41001_202004_D4_v00.nc

Once downloaded, data require extraction from the netCDF files. NetCDF files created before and after January 2011 have different structural formats, as outlined in Appendix B (pre-2011) and Appendix C (post-2011). Note that the datasets are inconsistent with respect to their reported variables over time, in both naming conventions and available parameters. Due to these netCDF format changes and inconsistent variable names, all variable data stored in the netCDF file should be extracted and merged with their corresponding date/time and GPS metadata. This ensures that all data in the netCDF files are captured, regardless of unexpected variable name changes and the addition of non-standard variables.

Another noteworthy issue in these netCDF data files is the inclusion of empty group variables (i.e., the netCDF creation process adds place markers for the standard suite of instruments but does not import data into those place markers if there are no data for that particular month). The possibility of group variables with no associated data needs to be accounted for to avoid batch import issues 
when reading in the data. For example, the Station 45001 June 2018 netCDF data file has an empty "payload 1" field, with no sub fields and no data in the "payload 2" sub fields for "air_temperature_sensor_1"; "anemomenter_1"; "anemomenter_2"; "barometer_1"; "barometer_2"; "gps_1"; or "ocean_temperature_sensor_1."

NDBC Data Concatenation. The NDBC text data files contain headers defining the data and units in each column. These text file headers have changed format over the years. For example, (a) the date and time nomenclature changed from YY to YYYY (in 1999), and back to YY (in 2007), and did not include minutes until 2005; (b) variable names have changed: wind direction nomenclature changed from WD to WDIR and atmospheric pressure from BAR to PRES. Additionally, the standard suite of meteorological variables has changed over the years (e.g., empty tide [TIDE] and visibility [VIS] fields are still present although those data have not been collected at buoy stations for many years). When concatenating NDBC data, be wary of redundant data (typically the beginning of each yearly file, where data from the end of the previous year are included in the following year's data file).

With technological advances, different wave instruments have been used since the 1970s, resulting in two different wave spectral frequency bands (38 and 47 frequency bands). The 38 frequencies are herewith considered as "old" frequencies, and the 47 frequency bands are considered as "new" frequency bands. In the latter case, the 47 frequency bands continue to be used today (even though they were the frequencies first used by NDBC from the 1970s until 1997-1998). Dates where these frequency bands changed occur non-uniformly from buoy to buoy as a result of servicing schedules and updated measurement requirements. Appendix D illustrates the format changes and the two frequency band ranges.

To handle these varying frequencies, create empty datasets with the 38 old and 47 new frequency bands and loop through each imported dataset to match each yearly or monthly dataset to the correct frequency bands. Using this method, no inconsistencies are detectable within the NDBC website data for the old and new frequency bands for all stations between 1970 and 2020 .

To save on storage space, NDBC-published directional variables, $r_{1}$ and $r_{2}$, that are scaled by 100 (NDBC 2018c). They require conversion to their correct units by multiplying them by 0.01 . Note that these NDBC data do not contain QC flags. Both the automated and manually-inserted NDBC QC flags have already been assessed and applied (where necessary) to the data by an NDBC MCC analyst before publishing.

NCEI Data Concatenation. Monthly NCEI netCDF files residing on the NCEI website contain all measured NDBC data. Combine each dataset with the associated date/time and GPS fields to create a dataset that matches the NDBC website file structure and nomenclature. During this concatenation step, be aware of duplicate data points with timestamps that are $\sim 5-10$ seconds apart.

Each netCDF file contains a significant amount of data with metadata that specifically describes each parameter. To read in only the relevant data, be aware of the generic character strings that are incorporated within the unique naming conventions, and result in duplicate date/time rows. For example, in addition to "wind_speed," there are "continuous_wind_speed"; "wind_speed_max_1"; "peak_wind_speed"; and "wind_speed_58" variables. Using only "wind_speed" will produce four additional rows of data initially intended for one. Additionally, 
the pre-2011 files have a "lon" variable name for GPS longitude, which also returns "solar_radiation_sensor_1_longwave_radiation". A number of these exception nomenclatures and variables are listed in Appendix E.

The post-2011 netCDF metadata fields contain multiple payloads (group variables) that store data from the various on-board sensor suites. Often, available secondary payload data are duplicates of the first set of payload data, which already contain any available primary and secondary sensor data and metadata.

Within the spectral data, there are multiple name extensions for $\mathrm{c}_{11}$ and $\mathrm{c}_{11 \mathrm{~m}}$ data (e.g., "i"; "k"; "l," historically defined as unique data sets for pre-netCDF file formatting). To recover the correct $\mathrm{c}_{11}$ data, select only "spectral_density_c"; “_k"; and "c $\mathrm{c}_{11}$ " data extensions (no "_i" or "c $11 \mathrm{~m}$ "). Likewise, for $\mathrm{c}_{11 \mathrm{~m}}$ data, select only " $\mathrm{c}_{11 \mathrm{~m}}$ " and " 1 " data extensions (no " $\mathrm{i}$ " or " $\mathrm{k}$ "). Important explanatory references to understand the netCDF data formats, units, record changes, etc., are recorded in the File Format Description for Meteorology, Oceanography, and Wave Spectra Data from Buoys (NCEI 2016) and the NOAA Marine Environmental Buoy Data (F291) Important Notices (NCEI 2014).

Standard meteorological netCDF data include two QC parameters for each variable: a "qc" and "release" flag field (spectral data do not have associated QC flags). The QC flags correspond to the NDBC flags as defined in the Handbook of Automated Data Quality Control Checks and Procedures, NDBC Technical Document 09-02 (NDBC 2009). If QC flags values are present, they should be applied to the variables to remove erroneous data (e.g., replace the data value with an "NA"). Once NDBC QC flags have been applied to the primary and secondary data, the secondary data may be used to augment the missing primary data. This will create a single, unique dataset for each variable (echoing the published NDBC website data, but with associated metadata). Note that there are exceptions that complicate the process. For example, the September 2012 netCDF data file for Station 41009 had 2334 continuous wind speed data values, 2334 QC flag values, but only 777 release flag values in the netCDF file instead of 2334.

To match the standard meteorological NDBC data, convert the NCEI netCDF air pressure from Pascal to Millibar (divide by 100), and air, water, and dew point temperatures from Kelvin to degrees Celsius (subtract 273.15). Remove any zero (“0”) wind gust values that are associated with NA wind speed values, as these wind gust data were either not flagged in the data file or included erroneously (i.e., it is not feasible to have gust data values when wind speed is missing).

When concatenating the wave spectral datasets, end-users will discover multiple, unusual frequency bands. Note that these unusual frequency bands are not present in the NDBC website data and are purely a manifestation of the NDBC netCDF creation process (see Appendix F for the Station 44014 non-matching frequency examples). As with the NDBC data, create empty datasets with the 38 old and 47 new frequency bands and loop through each imported dataset to match them to the correct frequency bands. This process ensures that no data are forced into incorrect frequency bands. When the "other" frequencies are present, save these as separate output files using a frequency count nomenclature, e.g. freq_48_cols, for ease of post-concatenation investigation.

NDBC and NCEI Data Comparisons. To determine which data to include in a "best available" NDBC dataset, both NDBC and NCEI datasets require comparisons. Exporting an automated table 
of start and end date/times for all equivalent NDBC and NCEI datasets (e.g., Table 3) allows for easy time period comparisons. These time comparison tables clearly highlight the differences between the two published data sources, considering that the matching data are collected from a single instrument. For example, Table 3 shows that for Station 46029, NDBC first published spectral wave energy, $\mathrm{c}_{11}$, on its website for Station 46029 on 08 January 1997 while the NCEI dataset includes an extra 4 days of data. More extreme differences are found in the Station 46029 spectral directional datasets: alpha 1 ; alpha $2 ; \mathrm{r}_{1}$; and $\mathrm{r}_{2}$, where NCEI datasets include additional years of data - NDBC alpha 2 data were only released to the public from the 07 May 1998 while the equivalent NCEI dataset contains data from 14 September 1995.

\begin{tabular}{|c|c|c|c|c|c|}
\hline NDBC Dataset & $\left.\begin{array}{c}\text { Start Time } \\
(\mathrm{mm} / \mathrm{dd} / \mathrm{yyyy})\end{array}\right)$ & $\begin{array}{l}\text { End Time } \\
(\mathrm{mm} / \mathrm{dd} / \mathrm{yyyy})\end{array}$ & NCEI Dataset & $\begin{array}{c}\text { Start Time } \\
(\mathrm{mm} / \mathrm{dd} / \mathrm{yyyy})\end{array}$ & $\begin{array}{l}\text { End Time } \\
(\mathrm{mm} / \mathrm{dd} / \mathrm{yyyy})\end{array}$ \\
\hline s_46029_ndbc_alpha1_freq_new & $\begin{array}{c}01 / 08 / 1997 \\
00: 00\end{array}$ & $\begin{array}{c}04 / 30 / 2020 \\
23: 00\end{array}$ & s_46029_ncei_alpha1_freq_new & $\begin{array}{c}01 / 04 / 1997 \\
19: 50\end{array}$ & $\begin{array}{c}04 / 30 / 2020 \\
23: 50\end{array}$ \\
\hline s_46029_ndbc_alpha1_freq_old & $\begin{array}{c}\text { 01/01/1996 } \\
00: 00\end{array}$ & $\begin{array}{c}12 / 03 / 2007 \\
08: 00\end{array}$ & s_46029_ncei_alpha1_freq_old & $\begin{array}{c}09 / 14 / 1995 \\
18: 50\end{array}$ & $\begin{array}{c}12 / 03 / 2007 \\
08: 50\end{array}$ \\
\hline s_46029_ndbc_alpha2_freq_new & $\begin{array}{c}11 / 20 / 1997 \\
00: 00\end{array}$ & $\begin{array}{c}04 / 30 / 2020 \\
23: 00 \\
\end{array}$ & s_46029_ncei_alpha2_freq_new & \begin{tabular}{|c|c|}
$01 / 04 / 1997$ \\
$19: 50$
\end{tabular} & $\begin{array}{c}04 / 30 / 2020 \\
23: 50\end{array}$ \\
\hline s_46029_ndbc_alpha2_freq_old & $\begin{array}{c}\text { 05/07/1998 } \\
00: 00\end{array}$ & $\begin{array}{c}12 / 03 / 2007 \\
08: 00\end{array}$ & s_46029_ncei_alpha2_freq_old & $\begin{array}{c}09 / 14 / 1995 \\
18: 50\end{array}$ & $\begin{array}{c}12 / 03 / 2007 \\
08: 50\end{array}$ \\
\hline s_46029_ndbc_c11_freq_new & $\begin{array}{c}01 / 08 / 1997 \\
00: 00\end{array}$ & $\begin{array}{c}04 / 30 / 2020 \\
23: 00\end{array}$ & s_46029_ncei_c11_freq_new & $\begin{array}{c}01 / 04 / 1997 \\
19: 50\end{array}$ & $\begin{array}{c}04 / 30 / 2020 \\
23: 50\end{array}$ \\
\hline s_46029_ndbc_c11_freq_old & $\begin{array}{c}01 / 01 / 1996 \\
00: 00\end{array}$ & $\begin{array}{c}12 / 03 / 2007 \\
08: 00\end{array}$ & s_46029_ncei_c11_freq_old & $\begin{array}{c}02 / 06 / 1986 \\
15: 00\end{array}$ & $\begin{array}{c}12 / 03 / 2007 \\
08: 50\end{array}$ \\
\hline s_46029_ndbc_r1_freq_new & $\begin{array}{c}11 / 20 / 1997 \\
00: 00\end{array}$ & $\begin{array}{c}04 / 30 / 2020 \\
23: 00\end{array}$ & s_46029_ncei_r1_freq_new & $\begin{array}{c}01 / 04 / 1997 \\
19: 50\end{array}$ & $\begin{array}{c}04 / 30 / 2020 \\
23: 50\end{array}$ \\
\hline s_46029_ndbc_r1_freq_old & $\begin{array}{c}05 / 07 / 1998 \\
00: 00\end{array}$ & $\begin{array}{c}12 / 03 / 2007 \\
08: 00\end{array}$ & s_46029_ncei_r1_freq_old & $\begin{array}{c}09 / 14 / 1995 \\
18: 50 \\
\end{array}$ & $\begin{array}{c}12 / 03 / 2007 \\
08: 50\end{array}$ \\
\hline s_46029_ndbc_r2_freq_new & $\begin{array}{c}11 / 20 / 1997 \\
00: 00\end{array}$ & $\begin{array}{c}04 / 30 / 2020 \\
23: 00 \\
\end{array}$ & s_46029_ncei_r2_freq_new & $\begin{array}{c}01 / 04 / 1997 \\
19: 50 \\
\end{array}$ & $\begin{array}{c}04 / 30 / 2020 \\
23: 50 \\
\end{array}$ \\
\hline s_46029_ndbc_r2_freq_old & $\begin{array}{c}05 / 07 / 1998 \\
00: 00 \\
\end{array}$ & $\begin{array}{c}12 / 03 / 2007 \\
08: 00 \\
\end{array}$ & s_46029_ncei_r2_freq_old & $\begin{array}{c}09 / 14 / 1995 \\
18: 50 \\
\end{array}$ & $\begin{array}{c}12 / 03 / 2007 \\
08: 50 \\
\end{array}$ \\
\hline s_46029_ndbc_stdmet & $\begin{array}{c}03 / 27 / 1984 \\
23: 00\end{array}$ & $\begin{array}{c}04 / 30 / 2020 \\
22: 50\end{array}$ & s_46029_ncei_stdmet & $\begin{array}{c}03 / 27 / 1984 \\
23: 00\end{array}$ & $\begin{array}{c}04 / 30 / 2020 \\
23: 50\end{array}$ \\
\hline
\end{tabular}

* "freq_new" suffix refers to the current used 47 frequency bands while "freq_old" suffix refers to the previously used 38 frequency bands.

This would appear to be bonus data until one realizes that the NDBC website contains only released data that are no longer experimental (i.e., they are no longer testing and modifying algorithms to process and QC the data). High data uncertainty is what prevented NDBC from releasing those data to the public. Using the additional NCEI data that have no associated flags to indicate unreliability requires extreme caution and a full understanding of the instrumentation, deployment metadata and processing algorithms applied to those data.

Matching dates/times. Data comparisons require matching on the nearest date and times. NDBC website wave and meteorological data were generally published on the hour while the NCEI data contain the more accurate, sensor reporting times. The NDBC wave sensors sample over a period of 20 minutes (minute $20-40$ of each hour) before transmitting its data to the on- 
board computing system, which packages the data and transmits to a shore receiver at 50 minutes past every hour. NDBC wave data are paired with hourly meteorological data and published to the Global Telecommunication System for immediate use, and after manual review, to the NDBC website. However, the delayed, monthly production of the netCDF data files allowed for the accurate sensor 50-minute time stamp to be applied to the variables.

The NDBC newly operational Self-Contained Ocean Observing Payload (SCOOP) (Hall et al. 2018b) allows for data transmission every 10 minutes, while wave data retain the hourly reporting rate. End-users should match these SCOOP data with their end goal in mind - whether to match to the hourly wave data (typically minute 40) and aggregate (average) or ignore the 10- to 50-minute data fields, or whether to merge the wave data with their corresponding hourly meteorological data fields and retain all the data. NDBC is in the process of migrating all buoy payloads to SCOOP systems during scheduled service visits.

Geographically clean. Of major importance is the use of available netCDF GPS metadata (post2011). As NDBC has, in the past, indiscriminately released data while the buoy is adrift or during service visits when the buoy is onboard a vessel, these GPS (latitude and longitude) metadata are invaluable to geographically clean these datasets.

Although buoy moorings generally retain the buoy within their specified watch circle (the area in which a buoy maintains its intended location), each buoy station has a different watch circle radius that is dependent on deployment water depths, mooring type, instrumentation, as well as wind and current effects. While it would be possible to interrogate the NDBC website to obtain the watch circle information for each buoy in the NDBC array, simply including all data within a radius of 1 degree around the mean deployment location is sufficient.

To capture the mean latitude and longitude positions and create a virtual watch circle, find the most common values from the floor and ceiling values of the tail of a sorted table of value occurrences (be sure to round off the latitude and longitude positions to at least three significant places to handle the multiple significant places available in the netCDF data). Note that simply calculating the mean latitude and longitude across the time period returns skewed results if the buoy was adrift for a long period of time. For example, the buoy at NDBC Station 42059 went adrift in 2009, 2013, 2014,2016 , and was vandalized twice in 2018. Isolating the mean latitude and longitude for those datasets returned a position that was nowhere near the original station location.

Quality control. Both the NDBC and NCEI variable data contain a few outliers that were not identified during NDBC QC protocols. Use the same sorted table methodology to test for and remove any erroneous outlier values in the datasets.

Best available datasets. The best available dataset for each NDBC station should consist of a combination of the geographically cleaned datasets created in the comparison steps above. Filter the NCEI netCDF geographically cleaned data to include only dates/time that match the NDBC manually quality-controlled data and filter the NDBC data to include only dates/times that match NCEI netCDF GPS positions within the virtual buoy watch circles.

Concatenate the filtered data for each NDBC station to include the following NDBC websitesourced, manually quality-controlled data that have been geographically cleaned and checked for 
outliers: significant wave height; dominant and peak wave periods; mean wave direction; wind direction; wind speed; wind gust; air pressure at sea level; air temperature; sea surface temperature; and wave spectral $\mathrm{c}_{11}$, alpha 1 , alpha $, \mathrm{r}_{1}, \mathrm{r}_{2}$. Add the filtered, geographical cleaned NCEI NDBC netCDF wave spectral datasets: $\mathrm{c}_{11 \mathrm{~m}}$, gamma, gamma $, \mathrm{Phi}_{\mathrm{h}}, \mathrm{R} / \mathrm{q}$ and sensor output data.

Comparison of best available NDBC datasets with Model/WIS data. Consistency between observational measurements and modeled wind and wave conditions is paramount to a successful evaluation. As the NDBC integral wave parameters, significant wave height, wave period, and wave direction, are based on their own mathematical definitions, so are those generated from the wave models used in the WIS hindcast. Wave spectra derived from the best available datasets produced by the aforementioned methods are the basis of the WIS evaluations. All wave parameters are computed via identical formulations found in the wave models used by WIS so that there is uniformity in the results. In addition, the frequency range found for each buoy dataset is used to set the WIS spectra so that the results are consistent with the buoy. WIS uses Fourier coefficients to compute the four directional spectral moments for each frequency: mean wave direction, spread, skewness, and kurtosis. Therefore, calculate these four Fourier directional parameters, defined as $a_{1}, b_{1}, a_{2}$, and $b_{2}$, from the alpha $a_{1}$, alpha $a_{2}, r_{1}$, and $r_{2}$ NDBC best available dataset as follows:

$$
\begin{aligned}
& a_{1}=-r_{1} \cdot \sin \left(\text { alpha }_{1} \cdot \pi / 180\right) \\
& b_{1}=-r_{1} \cdot \cos \left(\text { alpha }_{1} \cdot \pi / 180\right) \\
& a_{2}=-r_{2} \cdot \cos \left(2 \cdot \operatorname{alpha}_{2} \cdot \pi / 180\right) \\
& b_{2}=r_{2} \cdot \sin \left(2 \cdot \operatorname{alpha}_{2} \cdot \pi / 180\right)
\end{aligned}
$$

SUMMARY: This CHETN reviews the variations in NDBC data that are archived in two locations: on the NDBC website and in the official NOAA archives, which are located at the NCEI. This document contains multiple QC and processing suggestions that aim to improve the quality of these NDBC wave and meteorological data for use by the USACE and user community.

ADDITIONAL INFORMATION: This CHETN was prepared as part of the USACE Coastal and Ocean Data System (CODS) program by Candice Hall and Dr. Robert E. Jensen, U.S. Army Engineer Research and Development Center, Coastal and Hydraulics Laboratory, Coastal Processes Branch, Vicksburg, MS. Questions pertaining to this CHETN may be directed to Candice Hall (candice.hall@,usace.army.mil) or to the USACE CODS Program Manager, Dr. A. Spicer Bak.

This ERDC/CHL CHETN-I-?? should be cited as follows:

Hall, C., and R. E. Jensen. 2021. Utilizing Data from the NOAA National Data Buoy Center. ERDC/CHL CHETN-I-100. Vicksburg, MS: U.S. Army Engineer Research and Development Center. DOI: $\underline{\text { http://dx.doi.org/10.21079/11681/40059 }}$ 


\section{REFERENCES}

Crout, R. L., R. V. Hervey, and R.H. Bouchard. 2008. “Operational Field Test and Evaluation of NDBC'S Compact Ocean Observing System, Configured for Ocean Wave, Meteorological, and Ocean Current Profiling Measurements." Proc. 12th Conference on IOAS-AOLS, AMS. https://ams.confex.com/ams/88Annual/techprogram/paper 135170.htm

Earle, M. D. 2003. Nondirectional and Directional Wave Data Analysis Procedures. NDBC Technical Document 03-01. Silver Springs, MD: U.S. National Oceanic and Atmospheric Administration, National Weather Service, National Data Buoy Center.

Hall, C., R. Bouchard, R. Riley, R. Stewart, D. Wang, and S. DiNapoli. 2018a. Emerging National Data Buoy Center (NDBC) Wave Systems. Conference: JCOMM DBCP-34 Meeting, Cape Town, South Africa. https://www.researchgate.net/publication/328748840_Emerging_National_Data_Buoy_Center_NDBC_Wave_S ystems

Hall, C., R. H. Bouchard, and D. C. Petraitis. 2018b. Wave Module Field Evaluations between the NDBC's SCOOP on Modified 3-m Foam Hulls and Nearby Operational Systems. Conference: OCEANS '18 MTS/IEEE Charleston, SC. https://doi.org/10.1109/OCEANS.2018.8604855

Linzell, R. S. 2004. Analysis and Review of NDBC Wave Processing Algorithms and Software. Prepared by Neptune Science, Inc. for NDBC under NDBC Contract GS35F4807G. Silver Springs, MD: NOAA.

NCEI (National Center of Environmental Information). 2014. NOAA Marine Environmental Buoy Data (F291) Important Notices. U.S. National Oceanic and Atmospheric Administration, National Center of Environmental Information. Accessed 31 July 2020. https://www.nodc noaa.gov/BUOY/DOC/impnotes.html

NCEI. 2016. File Format Description for Meteorology, Oceanography, and Wave Spectra Data from Buoys (National Oceanographic Data Center F291 format). Silver Springs, MD: U.S. National Oceanic and Atmospheric Administration, National Center of Environmental Information. Accessed 31 July 2020. https://www.ncei.noaa.gov/data/oceans/nodc/formats/F291_NOAA_Marine_Environmental Buoy_Data.html

NCEI. 2020a. NOAA Marine Environmental Buoy Database. U.S. National Oceanic and Atmospheric Administration, National Center of Environmental Information. Accessed 31 July 2020. https://www.ncei.noaa.gov/access/marine-environmental-buoy-databasel

NCEI. 2020b. Information about the netCDF Data Files in the NOAA Marine Environmental Buoy Database. Accessed 31 July 2020. https://www.ncei.noaa.gov/access/marine-environmental-buoy-database/DOC/generalbuoy-netcdf.html

NDBC (National Data Buoy Center). 1996. Nondirectional and Directional Wave Data Analysis Procedure. NDBC Technical Document 96-01. Silver Springs, MD: U.S. National Oceanic and Atmospheric Administration, National Weather Service, National Data Buoy Center. -https://www.ndbc.noaa.gov/wavemeas.pdf

NDBC. 2003. Nondirectional and Directional Wave Data Analysis Procedure. NDBC Technical Document 03-01. Silver Springs, MD: U.S. National Oceanic and Atmospheric Administration, National Weather Service, National Data Buoy Center.

NDBC. 2009. Handbook of Automated Data Quality Control Checks and Procedures. NDBC Technical Document 09-02, Silver Springs. MD: U.S. National Oceanic and Atmospheric Administration, National Weather Service, National Data Buoy Center.

https://www.ndbc.noaa.gov/NDBCHandbookofAutomatedDataQualityControl2009.pdf 
NDBC. 2018a. How Are Significant Wave Height, Dominant Period, Average Period, and Wave Steepness Calculated? Silver Springs, MD: U.S. National Oceanic and Atmospheric Administration, National Weather Service, National Data Buoy Center. Accessed 01 August 2020. https://www.ndbc.noaa.gov/wavecalc.shtml

NDBC. 2018b. How Are Spectral Wave Data Derived from Buoy Motion Measurements? Silver Springs, MD: U.S. National Oceanic and Atmospheric Administration, National Weather Service, National Data Buoy Center. Accessed 01 August 2020. https://www.ndbc.noaa.gov/wave.shtml

NDBC. 2018c. Measurement Descriptions and Units. Silver Springs, MD: U.S. National Oceanic and Atmospheric Administration, National Weather Service, National Data Buoy Center. Accessed 04 August 2020. https://www.ndbc.noaa.gov/measdes.shtml

NDBC. 2020. Station 46029 (LLNR 688) - COLUMBIA RIVER BAR - 20NM West of Columbia River Mouth. Silver Springs, MD: U.S. National Oceanic and Atmospheric Administration, National Weather Service, National Data Buoy Center. Accessed 28 July 2020. https://www.ndbc.noaa.gov/station_page.php?station=46029

Ortiz-Royero, J. C., and A. Mercado-Irizarry. 2008. "An Intercomparison of SWAN and WAVEWATCH III Models with Data from NDBC-NOAA Buoys at Oceanic Scales.” Coastal Engineering Journal 50(01): 47-73 https://doi.org/10.1142/S0578563408001739

Reguero, B. G., M. Menéndez, F. J. Méndez, R. Mínguez, and I. J. Losada. 2012. “A Global Ocean Wave (GOW) Calibrated Reanalysis from 1948 Onwards." Coastal Engineering 65: 38-55. https://doi.org/10.1016/j.coastaleng.2012.03.003

Riley, R., C. Teng, R. Bouchard, R. Dinoso, and T. Mettlach. 2011. "Enhancements to NDBC's Digital Directional Wave Module." Conference: OCEANS'11 MTS/IEEE KONA, Waikoloa, HI, 2011. https://doi.org/10.23919/OCEANS.2011.6107025

Riley, R., and R. H. Bouchard. 2015. "An Accuracy Statement for the Buoy Heading Component of NDBC Directional Wave Measurements." Conference: The Twenty-fifth (2015) International Ocean and Polar Engineering Conference, Kona, Big Island, Hawaii.

Riley, R., C. Hall, R. Stewart, S. DiNapoli, and D. W. Wang. 2019. "NDBC OWL Wave System Development." Conference: 2019 IEEE/OES Twelfth Currents, Waves, Turbulence Measurements and Applications Workshop (CWTMA). https://doi.org/10.1109/CWTM43797.2019.8955248

Rusu, L., and C. Guedes Soares. 2012. "Wave Energy Assessments in the Azores islands." Renewable Energy 45: 183-196. https://doi.org/10.1016/j.renene.2012.02.027

Steele, K. E., J. C. Lau, and Y. L. Hsu. 1985. "Theory and Application of Calibration Techniques for an NDBC Directional Wave Measurements Buoy." IEEE Journal of Oceanic OE-10(4). https://ieeexplore.ieee.org/stamp/stamp.jsp? arnumber $=1145116$

Stopa, J. E., and K. F. Cheung. 2014. "Intercomparison of Wind and Wave Data from the ECMWF Reanalysis Interim and the NCEP Climate Forecast System Reanalysis." Ocean Modelling 75: 65-83. https://doi.org/10.1016/j.ocemod.2013.12.006

Stopa, J. E., and A. Mouche. 2016. "Significant Wave Heights from Sentinel-1 SAR: Validation and Applications." Journal of Geophysical Research 122(3). https://doi.org/10.1002/2016JC012364

Teng, C., and G. L. Timpe. 1995. "Field Evaluation of the Value-Engineered 3-meter Discus Buoy." Conference Proceedings: Challenges of Our Changing Global Environment, OCEANS '95 MTS/IEEE, San Diego, California, USA, 1995, Vol. 1., 316-322. https://doi: 10.1109/OCEANS.1995.526789

Teng, C., T. Mettlach, J. Chaffin, R. Bass, C. Bond, C. Carpenter, R. Dinoso, M. Hellenschmidt, and L. Bernard. 2007. "National Data Buoy Center 1.8-meter Discus Buoy, Directional Wave System." Proc. MTS/IEEE Oceans 2007 Conference, Vancouver, Canada. https://ieeexplore.ieee.org/stamp/stamp.jsp?tp $=\&$ arnumber $=4449298$ 
Teng, C., R. Bouchard, R. Riley, T. Mettlach, R. Dinoso, and J. Chaffin, 2009. "NDBC's Digital Directional Wave Module." OCEANS 2009, Biloxi, MS, 1-8. https://doi.org/10.23919/OCEANS.2009.5422386

USACE (U.S. Army Corps of Engineers). 2002. U.S. Army Corps of Engineers Coastal Engineering Manual - Part II. CECW-CE EM1110-2-1100. Washington, DC: USACE. https://www.publications.usace.army.mil/LinkClick.aspx?fileticket $=$ BL4oDlcbwvc\%3d\&tabid $=16439 \&$ portalid $=76 \&$ mid $=43544$

USACE. 2020. Wave Information Studies. Accessed 26 June 2020. http://wis.usace.army.mil/; http://wis.usace.army.mil/wis documentation.html

Van Nieuwkoop, J. C. C., H. C. M. Smith, G. H. Smith, and L. Johanning. 2013. "Wave Resource Assessment along the Cornish Coast (UK) from a 23-year Hindcast Dataset Validated against Buoy Measurements." Renewable Energy 58(1-14). http://dx.doi.org/10.1016/j.renene.2013.02.033

NOTE: The contents of this technical note are not to be used for advertising, publication, or promotional purposes. Citation of trade names does not constitute an official endorsement or approval of the use of such products. 


\section{Appendix A: NDBC Conversion Algorithms and Data Quality Assurance Parameters}

\section{c11 to $\mathbf{c}_{11 \mathrm{~m}}$}

The conversion algorithm (NDBC 2003) to calculate wave displacement spectral density $\mathrm{c}_{11}\left[\mathrm{~m}^{2} / \mathrm{Hz}\right]$ from wave acceleration spectral density $\mathrm{c}_{11 \mathrm{~m}}\left(\mathrm{~m} / \mathrm{s}^{2}\right)^{2} / \mathrm{Hz}$ is (as derived from Steele et al. 1985):

$$
c_{11}(f)=c_{11 m}(f) /\left(R_{s H}(f)\right)^{2}
$$

If hull response correction $\left(\mathrm{R}_{\mathrm{hH}}\right)$ is assumed insignificant (i.e., a perfect wave following buoy where $\mathrm{R}_{\mathrm{hH}}=1$ ):

$$
c_{11}=c_{11 m} /(2 \pi(f))^{4}
$$

phi $_{h}$

NDBC uses angle phi $(f)$ to correctly determine the mean and principle wave directions ( $\operatorname{alpha}_{1}(f)$ and $\operatorname{alpha}_{2}(f)$ respectively) in accelerometer-based directional wave systems. Equations (36) and (37) in Steele et al. (1985) are adapted by NDBC (Linzell 2004, 6-7) for each frequency band as

$$
p h i(f)=p h i_{h}(f)+p h i_{s H}(f)=p h i_{h}(f)+\pi
$$

In which

$$
p h i_{h}(f)=p h i_{h H}(f)-p h i_{h S}(f)
$$

where the acceleration sensor $\left(_{\mathrm{s}}\right)$ heave $\left(_{\mathrm{H}}\right)$ phase lag angle is represented as $\mathrm{phi}_{\mathrm{sH}}(f)$; the phase lag angle of the hull heave $(\mathrm{h})$ is represented as $\mathrm{phi}_{\mathrm{hH}}(f)$, and the phase lag of the hull slope $(\mathrm{s})$ is represented as phi $\mathrm{h}_{\mathrm{h}}(f)$.

$\mathbf{R}^{\mathrm{h}} / \mathbf{q}$

NDBC estimates frequency dependent sensor and hull-mooring responses (including wave depth effects). Removing the known sensor effects (static over time), allows NDBC to investigate the hull amplitude response, $R^{h}$ (Earle 2003). To separate the responses, the parameter, $q$ is defined as (Earle 2003):

$$
q=\tanh (k d)=\frac{\omega^{2}}{g k}
$$

where $k$ is wave number, $d$ is mean wave depth, $g$ is acceleration due to gravity and $\omega$ is the perpendicular angular velocity component. Where $q=1$ in deep water, and canceling out $k$, yields

what NDBC actually uses:

$$
\frac{R^{h}}{q}=g \sqrt{\frac{C_{22}^{m}+C_{33}^{m}}{C_{11}^{m}}}
$$

From the original equation: $\quad \frac{R^{h}}{q}=\left(\frac{g R^{s H}}{\omega^{2}}\right)\left[\frac{\left(C_{22}^{m}+C_{33}^{m}\right)}{\left(C_{11}^{m}\right)}\right]^{\frac{1}{2}}$

\section{gamma $_{2}$ and gamma 3}

These parameters allow the on-board wave sensor to determine whether values at each frequency are based on noise or whether waves are present at that direction. This is necessary as the buoy hull delays wave heave and wave slope at the same frequencies, but by different amounts. The differences in these two delay angles are calculated per hour for each frequency. To correct for these angles' deviation from zero, alternations are made the co- and quad-spectra, $C_{1 j}$ and $Q_{1 j}$, to ensure that $Q_{1 j}$ contains all the information needed to calculate wave direction. However, to help the computer code identify whether these frequency differences are just noise (i.e., no waves at that particular direction) the software performs statistical checks as follows:

$$
\begin{array}{cc}
\text { For normal waves: if } & \operatorname{abs}\left(Q_{1 j}\right) \gg \operatorname{abs}\left(C_{1 j}\right) \\
\text { and if } & \arctan \left(\left(C_{1 j}\right) /\left(Q_{1 j}\right)\right)
\end{array}
$$

returns a small angle $\left(<5^{\circ}\right)$, waves, not noise, are present at that frequency. 


\section{Appendix B: NCEI-sourced NDBC netCDF data format - pre 2011}

\begin{tabular}{|c|c|c|}
\hline Name & Long Name & Type \\
\hline 7 46029_201012.nc & Meteorological and oceanographic data collected from a Nati... & Local File \\
\hline$\vartheta$ air_pressure_at_sea_level & air pressure at sea level & $1 \mathrm{D}$ \\
\hline$\checkmark$ air_temperature & air temperature & $1 \mathrm{D}$ \\
\hline$\vartheta$ alpha1 & mean wave direction at specified frequency & $2 \mathrm{D}$ \\
\hline$\vartheta$ alpha2 & principal wave direction at specified frequency & $2 \mathrm{D}$ \\
\hline$\vartheta$ anemometer_height & anemometer height & $1 \mathrm{D}$ \\
\hline$\vartheta$ average_wave_period & sea surface wave mean period from variance spectral densit... & $1 \mathrm{D}$ \\
\hline$\vartheta$ bottom_depth & sea floor depth below sea level & $1 \mathrm{D}$ \\
\hline$\theta c 11 \_k$ & sea surface wave variance spectral density & $2 \mathrm{D}$ \\
\hline$\theta$ C11_ & sea surface wave directional variance spectral density c11 u... & $2 \mathrm{D}$ \\
\hline$\theta \mathrm{C} 12 \_$ & sea surface wave directional variance spectral density $c 12$ u... & $2 \mathrm{D}$ \\
\hline$\theta \mathrm{C} 13 \_$ & sea surface wave directional variance spectral density c13 u... & $2 \mathrm{D}$ \\
\hline$\theta \mathrm{C} 22 \mathrm{I}$ & sea surface wave directional variance spectral density c $22 \mathrm{u} .$. & $2 \mathrm{D}$ \\
\hline$\theta \mathrm{C} 23 \_$ & sea surface wave directional variance spectral density c $23 \mathrm{u} . .$. & $2 \mathrm{D}$ \\
\hline ○ C33_ & sea surface wave directional variance spectral density $c 33 \mathrm{u} . .$. & $2 \mathrm{D}$ \\
\hline$\ominus$ continuous_wind_direction & continuous wind direction & $1 \mathrm{D}$ \\
\hline$\vartheta$ continuous_wind_speed & continuous wind speed & $1 \mathrm{D}$ \\
\hline$\vartheta$ direction_of_hourly_max_gust & direction of hourly max gust & $1 \mathrm{D}$ \\
\hline$\vartheta$ dominant_wave_period & sea surface wave period at variance spectral density maximum & $1 \mathrm{D}$ \\
\hline$\vartheta$ end_of_wave_data_acquisition_k & end of wave data acquisition time & $1 \mathrm{D}$ \\
\hline$\vartheta$ hourly_max_gust & hourly max gust & $1 \mathrm{D}$ \\
\hline$\ominus$ lat & latitude & - \\
\hline$\vartheta$ lon & longitude & - \\
\hline$\vartheta$ magnetic_variation & magnetic variation from north & $1 \mathrm{D}$ \\
\hline$\checkmark$ mean_wave_direction & sea surface wave from direction & $1 \mathrm{D}$ \\
\hline Q Q12_ & sea surface wave directional variance spectral density q12 u... & $2 \mathrm{D}$ \\
\hline Q Q13_ & sea surface wave directional variance spectral density q13 u... & $2 \mathrm{D}$ \\
\hline$\theta r 1$ & first normalized polar coordinate of the Fourier coefficients & $2 \mathrm{D}$ \\
\hline$\vartheta \mathrm{r} 2$ & second normalized polar coordinate of the Fourier coefficients & $2 \mathrm{D}$ \\
\hline$\vartheta$ sampling_duration_waves & wave sampling duration in minute & $1 \mathrm{D}$ \\
\hline ? sampling_rates_waves & wave sampling rate per minute & $1 \mathrm{D}$ \\
\hline$\vartheta$ sea_surface_temperature & sea surface temperature & $1 \mathrm{D}$ \\
\hline sensor_output & sensor output type & $1 \mathrm{D}$ \\
\hline$\checkmark$ significant_wave_height & sea surface wave significant height & $1 \mathrm{D}$ \\
\hline$\vartheta$ speed_averaging_method & speed averaging method & $1 \mathrm{D}$ \\
\hline$\vartheta$ standard_deviation_of_hourly_speed & standard deviation of hourly speed & $1 \mathrm{D}$ \\
\hline$\vartheta$ station & Unique identifier for each feature instance & - \\
\hline$\theta$ time & time & $1 \mathrm{D}$ \\
\hline$\checkmark$ time10 & ten minute time & $1 \mathrm{D}$ \\
\hline$\vartheta$ time10_bnds & ten minute time bounds & $2 \mathrm{D}$ \\
\hline$\vartheta$ time_bnds & time bounds & $2 \mathrm{D}$ \\
\hline$\vartheta$ time_wpm_20 & twenty minute time & $1 \mathrm{D}$ \\
\hline$\odot$ time_wpm_20_bnds & twenty minute time bounds & $2 \mathrm{D}$ \\
\hline$\vartheta$ timem & max hourly measured time & $1 \mathrm{D}$ \\
\hline$\ominus$ timem_bnds & max hourly measured time bounds & $2 \mathrm{D}$ \\
\hline$\vartheta$ total_intervals_waves & frequency interval count & $1 \mathrm{D}$ \\
\hline ? wave_wpm & sea surface wave frequency & $1 \mathrm{D}$ \\
\hline$\vartheta$ wave_wpm_bnds & sea surface wave frequency bounds & $2 \mathrm{D}$ \\
\hline$\vartheta$ wind_direction & average wind direction & $1 \mathrm{D}$ \\
\hline$\theta$ wind_gust & wind speed of gust & $1 \mathrm{D}$ \\
\hline$\checkmark$ wind_gust_averaging_period & wind gust averaging period in seconds & $1 \mathrm{D}$ \\
\hline$\bullet$ wind_sampling_duration & wind sampling duration in minute & $1 \mathrm{D}$ \\
\hline$\theta$ wind_speed & average wind speed & $1 \mathrm{D}$ \\
\hline
\end{tabular}




\begin{tabular}{|c|c|c|}
\hline Name & Long Name & Type \\
\hline 746029_202001_D7.nc & Meteorological and Oceanographic Data Collected from the... & Local File \\
\hline$\nabla$ QPayload_1 & payload_1 & - \\
\hline$\nabla$ Qair_temperature_sensor_1 & payload_1/air_temperature_sensor_1 & - \\
\hline$\vartheta$ air_temperature & air temperature & GeoTraj \\
\hline$\checkmark$ air_temperature_detail_qc & air temperature detail qc & - \\
\hline air_temperature_qc & air temperature qc & GeoTraj \\
\hline$\checkmark$ air_temperature_release & air temperature release & GeoTraj \\
\hline$\checkmark$ dew_point_temperature & dew point temperature & GeoTraj \\
\hline$\checkmark$ dew_point_temperature_detail_qc & dew point temperature detail qc & - \\
\hline$\checkmark$ dew_point_temperature_qc & dew point temperature qc & GeoTraj \\
\hline$\checkmark$ dew_point_temperature_release & dew point temperature release & GeoTraj \\
\hline Q air_temperature_sensor_2 & payload_1/air_temperature_sensor_2 & - \\
\hline$\nabla$ Qanemometer_1 & payload_1/anemometer_1 & - \\
\hline$\vartheta$ continuous_wind_direction & wind from direction & $1 \mathrm{D}$ \\
\hline$\checkmark$ continuous_wind_direction_detail_qc & continuous wind direction detail qc & - \\
\hline$\odot$ continuous_wind_direction_qc & continuous wind direction qc & $1 \mathrm{D}$ \\
\hline$\checkmark$ continuous_wind_direction_release & continuous wind direction release & GeoTraj \\
\hline$\odot$ continuous_wind_speed & wind speed & $1 \mathrm{D}$ \\
\hline$\checkmark$ continuous_wind_speed_detail_qc & continuous wind speed detail qc & - \\
\hline$\vartheta$ continuous_wind_speed_qc & continuous wind speed qc & $1 \mathrm{D}$ \\
\hline$\checkmark$ continuous_wind_speed_release & continuous wind speed release & GeoTraj \\
\hline ○ direction_of_hourly_max_gust & wind from direction & $1 \mathrm{D}$ \\
\hline$\checkmark$ direction_of_hourly_max_gust_detail_qc & wind from direction detail qc & - \\
\hline$\odot$ direction_of_hourly_max_gust_qc & wind from direction qc & $1 \mathrm{D}$ \\
\hline$\checkmark$ direction_of_hourly_max_gust_release & direction of hourly max gust release & GeoTraj \\
\hline$\checkmark$ direction_of_max_1_minute_wind_speed & wind from direction & $1 \mathrm{D}$ \\
\hline$\checkmark$ direction_of_max_1_minute_wind_spee... & wind from direction detail qc & - \\
\hline$\checkmark$ direction_of_max_1_minute_wind_spee... & wind from direction qc & $1 \mathrm{D}$ \\
\hline$\checkmark$ direction_of_max_1_minute_wind_spee... & direction of max 1 minute wind speed release & GeoTraj \\
\hline$\odot$ hourly_max_gust & wind speed of gust & $1 \mathrm{D}$ \\
\hline$\ominus$ hourly_max_gust_detail_qc & wind speed of gust detail qc & - \\
\hline hourly_max_gust_qc & wind speed of gust qc & $1 \mathrm{D}$ \\
\hline$\vartheta$ hourly_max_gust_release & hourly max gust release & GeoTraj \\
\hline$\checkmark$ max_1_minute_wind_speed & wind speed & $1 \mathrm{D}$ \\
\hline$\checkmark$ max_1_minute_wind_speed_detail_qc & wind speed detail qc & - \\
\hline ? max_1_minute_wind_speed_qc & wind speed qc & $1 \mathrm{D}$ \\
\hline$\checkmark$ max_1_minute_wind_speed_release & max 1 minute wind speed release & GeoTraj \\
\hline$\odot$ wind_direction & wind from direction & GeoTraj \\
\hline$\checkmark$ wind_direction_detail_qc & wind direction detail qc & - \\
\hline$\odot$ wind_direction_qc & wind direction qc & GeoTraj \\
\hline$\checkmark$ wind_direction_release & wind direction release & GeoTraj \\
\hline$\checkmark$ wind_gust & wind speed of gust & GeoTraj \\
\hline$\checkmark$ wind_gust_detail_qc & wind gust detail qc & - \\
\hline
\end{tabular}


ERDC/CHL CHETN-I-100

March 2021

\begin{tabular}{|c|c|c|c|}
\hline & Name & Long Name & Type \\
\hline & $\ominus$ wind_speed_qc & wind speed qc & GeoTraj \\
\hline & $\checkmark$ wind_speed_release & wind speed release & GeoTraj \\
\hline$\triangleright$ & Q anemometer_2 & payload_1/anemometer_2 & - \\
\hline \multicolumn{2}{|r|}{ V Qbarometer_1 } & payload_1/barometer_1 & - \\
\hline & $\vartheta$ air_pressure & air pressure & GeoTraj \\
\hline & $\diamond$ air_pressure_at_sea_level & air pressure at sea level & $1 \mathrm{D}$ \\
\hline & ○ air_pressure_at_sea_level_detail_qc & air pressure at sea level detail qc & - \\
\hline & $\checkmark$ air_pressure_at_sea_level_qc & air pressure at sea level qc & GeoTraj \\
\hline & $\checkmark$ air_pressure_at_sea_level_release & air pressure at sea level release & GeoTraj \\
\hline & air_pressure_detail_qc & air pressure detail qc & - \\
\hline & air_pressure_qc & air pressure qc & GeoTraj \\
\hline & $\vartheta$ air_pressure_release & air pressure release & GeoTraj \\
\hline & Qbarometer_2 & payload_1/barometer_2 & - \\
\hline \multicolumn{2}{|r|}{$\nabla$ Qgps_1 } & payload_1/gps_1 & - \\
\hline & latitude & latitude & $1 \mathrm{D}$ \\
\hline & $\odot$ latitude_detail_qc & latitude detail qc & - \\
\hline & $\odot$ latitude_qc & latitude qc & GeoTraj \\
\hline & O latitude_release & latitude release & GeoTraj \\
\hline & $\odot$ longitude & longitude & $1 \mathrm{D}$ \\
\hline & S longitude_detail_qc & longitude detail qc & - \\
\hline & $\checkmark$ longitude_qc & longitude qc & GeoTraj \\
\hline & $\checkmark$ longitude_release & longitude release & GeoTraj \\
\hline \multicolumn{2}{|r|}{ V Q 2 humidity_sensor_1 } & payload_1/humidity_sensor_1 & - \\
\hline & $\checkmark$ relative_humidity & relative humidity & GeoTraj \\
\hline & $\checkmark$ relative_humidity_detail_qc & relative humidity detail qc & - \\
\hline & $\checkmark$ relative_humidity_qc & relative humidity qc & GeoTraj \\
\hline & $\checkmark$ relative_humidity_release & relative humidity release & GeoTraj \\
\hline \multicolumn{2}{|r|}{ Q Q ocean_temperature_sensor_1 } & payload_1/ocean_temperature_sensor_1 & - \\
\hline & sea_surface_temperature & sea surface temperature & GeoTraj \\
\hline & $\checkmark$ sea_surface_temperature_detail_qc & sea surface temperature detail qc & - \\
\hline & sea_surface_temperature_qc & sea surface temperature qc & GeoTraj \\
\hline & S sea_surface_temperature_release & sea surface temperature release & GeoTraj \\
\hline \multicolumn{2}{|r|}{$\nabla$ Gave_sensor_1 } & payload_1/wave_sensor_1 & - \\
\hline & $\checkmark$ alpha1 & mean wave direction at specified frequency & $2 \mathrm{D}$ \\
\hline & $\checkmark$ alpha2 & principal wave direction at specified frequency & $2 \mathrm{D}$ \\
\hline & $\checkmark$ average_period & sea surface wave mean period from variance spectral dens... & $1 \mathrm{D}$ \\
\hline & average_period_detail_qc & sea surface wave mean period from variance spectral dens... & - \\
\hline & $\checkmark$ average_period_qc & sea surface wave mean period from variance spectral dens... & $1 \mathrm{D}$ \\
\hline & $\odot$ average_period_release & average period release & GeoTraj \\
\hline & O c11 & sea surface wave variance spectral density & $2 \mathrm{D}$ \\
\hline & $\vartheta c 11 \mathrm{~m}$ & sea surface wave variance spectral density uncorrected & $2 \mathrm{D}$ \\
\hline & $\checkmark$ dominant_period & sea surface wave period at variance spectral density maxi... & $1 \mathrm{D}$ \\
\hline & $\checkmark$ dominant_period_detail_qc & sea surface wave period at variance spectral density maxi... & - \\
\hline & $\checkmark$ dominant_period_qc & sea surface wave period at variance spectral density maxi... & $1 \mathrm{D}$ \\
\hline & $\checkmark$ dominant_period_release & dominant period release & GeoTraj \\
\hline & $\checkmark$ gamma2 & gamma 2 coefficient for quad spectra & $2 \mathrm{D}$ \\
\hline & gamma3 & gamma3 coefficient for quad spectra & $2 \mathrm{D}$ \\
\hline & $\checkmark$ mean_wave_direction & sea surface wave from direction & $1 \mathrm{D}$ \\
\hline & mean_wave_direction_detail_qc & sea surface wave from direction detail qc & - \\
\hline & mean_wave_direction_qc & sea surface wave from direction qc & $1 \mathrm{D}$ \\
\hline & $\checkmark$ mean_wave_direction_release & mean wave direction release & GeoTraj \\
\hline
\end{tabular}




\begin{tabular}{|c|c|c|c|}
\hline \multicolumn{2}{|r|}{ Name } & Long Name & Type \\
\hline \multicolumn{2}{|r|}{$\vartheta$ phih } & phih coefficient for quad spectra & $2 \mathrm{D}$ \\
\hline \multicolumn{2}{|r|}{$\theta \mathrm{r} 1$} & first normalized polar coordinate of the Fourier coefficients & $2 \mathrm{D}$ \\
\hline \multicolumn{2}{|r|}{$\theta \mathrm{r} 2$} & second normalized polar coordinate of the Fourier coefficients & $2 \mathrm{D}$ \\
\hline \multicolumn{2}{|r|}{$\theta$ rhq } & rhq coefficient for quad spectra & $2 \mathrm{D}$ \\
\hline \multicolumn{2}{|r|}{$\checkmark$ significant_wave_height } & sea surface wave significant height & $1 \mathrm{D}$ \\
\hline \multicolumn{2}{|r|}{$\bullet$ significant_wave_height_detail_qc } & sea surface wave significant height detail qc & - \\
\hline \multicolumn{2}{|r|}{ significant_wave_height_qc } & sea surface wave significant height qc & $1 \mathrm{D}$ \\
\hline \multicolumn{2}{|r|}{$\theta$ significant_wave_height_release } & significant wave height release & GeoTraj \\
\hline & Opayload_2 & payload_2 & - \\
\hline & Q Qair_temperature_sensor_1 & payload_2/air_temperature_sensor_1 & - \\
\hline & Dair_temperature_sensor_2 & payload_2/air_temperature_sensor_2 & - \\
\hline & - anemometer_1 & payload_2/anemometer_1 & - \\
\hline & Q 2 anemometer_2 & payload_2/anemometer_2 & - \\
\hline & Sbarometer_1 & payload_2/barometer_1 & - \\
\hline & Q barometer_2 & payload_2/barometer_2 & - \\
\hline & Ogps_1 & payload_2/gps_1 & - \\
\hline & Q Q humidity_sensor_1 & payload_2/humidity_sensor_1 & - \\
\hline & Q Q Oocean_temperature_sensor_1 & payload_2/ocean_temperature_sensor_1 & - \\
\hline & Qlwave_sensor_1 & payload_2/wave_sensor_1 & - \\
\hline & $\vartheta$ time & time & $1 \mathrm{D}$ \\
\hline & $\checkmark$ time 10 & ten minute time & $1 \mathrm{D}$ \\
\hline & $\vartheta$ time_wpm_20 & time & $1 \mathrm{D}$ \\
\hline & $\checkmark$ timem & max hourly measured time & $1 \mathrm{D}$ \\
\hline & $\theta$ wave_wpm & sea surface wave frequency & $1 \mathrm{D}$ \\
\hline & S wave_wpm_bnds & wave wpm bnds & $2 \mathrm{D}$ \\
\hline
\end{tabular}




\section{Appendix D: NDBC standard meteorological and wave spectra text file formats: $1970-2020$}

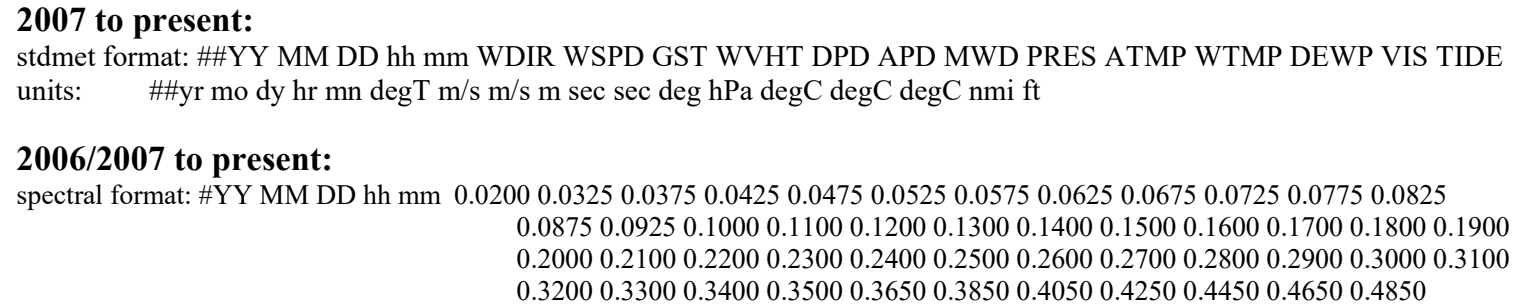

2005 and 2006: - skip first line, has no skip (\#) flag stdmet format: YYYY MM DD hh mm WD WSPD GST WVHT DPD APD MWD BAR ATMP WTMP DEWP VIS TIDE units: no units in files

\section{5/2006:}

spectral format: YYYY MM DD hh mm

0.0300 .0400 .0500 .0600 .0700 .0800 .0900 .1000 .1100 .1200 .1300 .1400 .150

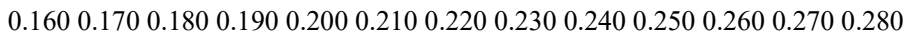

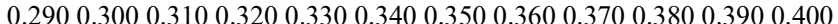

2000 to 2004: - no minute column - don't skip lines, missing tide data in some sets stdmet data format: YYYY MM DD hh WD WSPD GST WVHT DPD APD MWD BAR ATMP WTMP DEWP VIS TIDE units: no units in files

spectral data format: YYYY MM DD hh

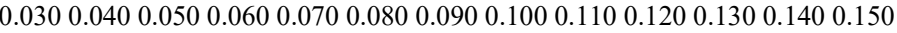

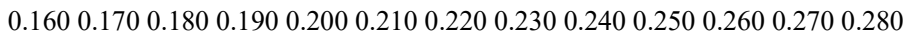

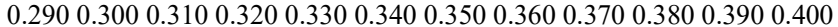

1999: - no TIDE or minute column

stdmet data format: YYYY MM DD hh WD WSPD GST WVHT DPD APD MWD BAR ATMP WTMP DEWP VIS units: no units in files

spectral data format: YYYY MM DD hh

0.0300 .0400 .0500 .0600 .0700 .0800 .0900 .1000 .1100 .1200 .1300 .1400 .150 0.1600 .1700 .1800 .1900 .2000 .2100 .2200 .2300 .2400 .2500 .2600 .2700 .280

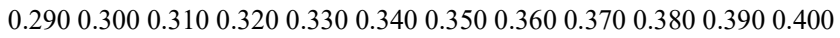

1970s to 1998: - no TIDE or minute column, note year and spectral frequency change stdmet data format: YY MM DD hh WD WSPD GST WVHT DPD APD MWD BAR ATMP WTMP DEWP VIS units: no units in files

1970s to $1997 / 1998$ :

spectral data format: YY MM DD hh $\quad 0.02000 .03250 .03750 .04250 .04750 .05250 .05750 .06250 .06750 .07250 .07750 .0825$ 0.08750 .09250 .10000 .11000 .12000 .13000 .14000 .15000 .16000 .17000 .18000 .1900 0.20000 .21000 .22000 .23000 .24000 .25000 .26000 .27000 .28000 .29000 .30000 .3100 $\begin{array}{lllllllllll}0.3200 & 0.3300 & 0.3400 & 0.3500 & 0.3650 & 0.3850 & 0.4050 & 0.4250 & 0.4450 & 0.4650 & 0.4850\end{array}$ 


\section{Appendix E: NCEI NDBC netCDF File Nomenclature Exceptions}

Remove these nomenclatures from data selection for each as they create duplicate date/time rows that bias comparisons.

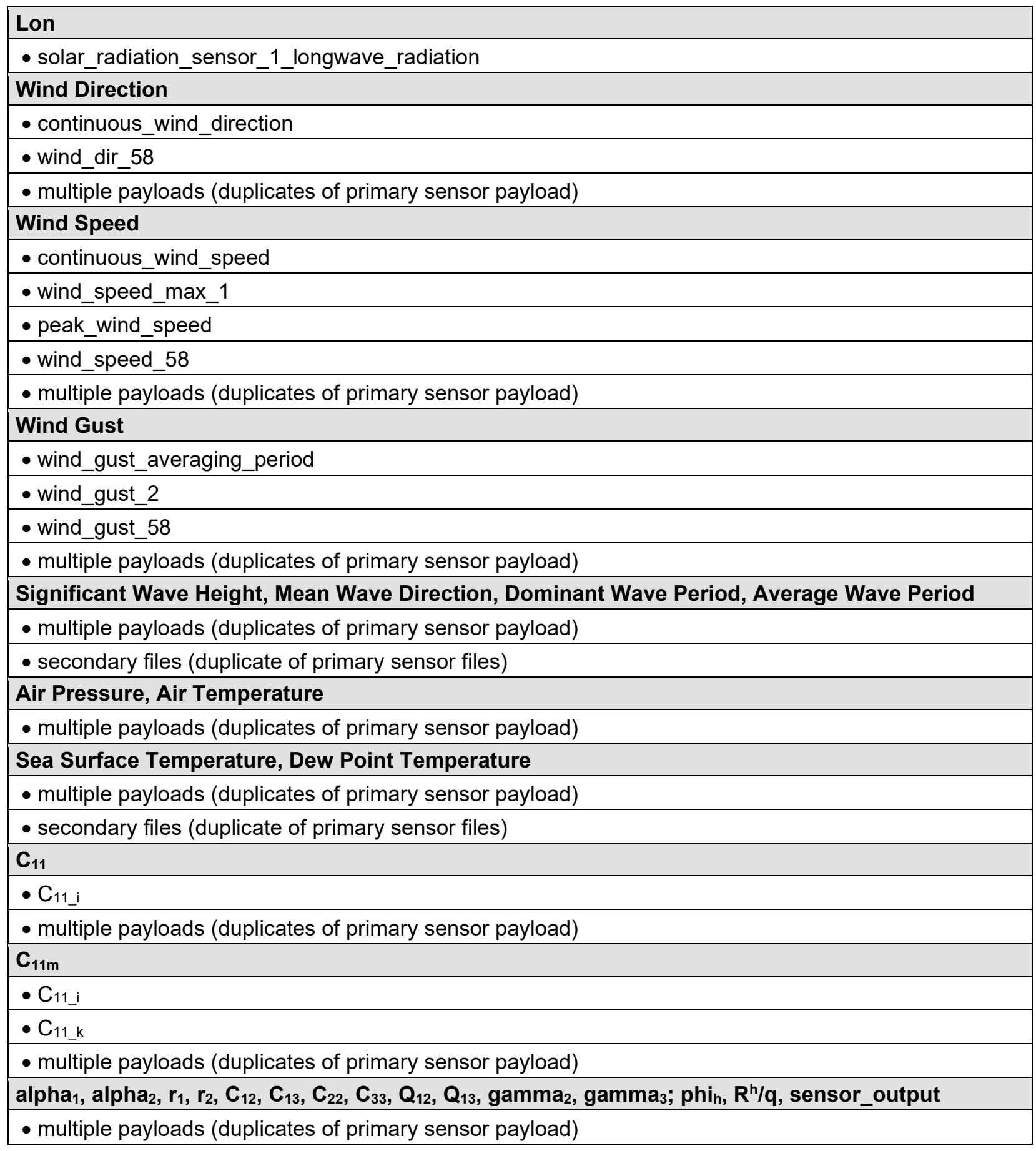


ERDC/CHL CHETN-I-100

March 2021

\section{Appendix F: NDBC Station 44014}

NDBC central spectral frequencies vary over time with the use of different wave sensors: 1996/01-1997/03.

\begin{tabular}{|l|l|l|l|l|l|l|l|l|l|l|l|}
\hline 0.02 & 0.0325 & 0.0375 & 0.0425 & 0.0475 & 0.0525 & 0.0575 & 0.0625 & 0.0675 & 0.0725 & 0.0775 & 0.0825 \\
\hline 0.0875 & 0.0925 & 0.1 & 0.11 & 0.12 & 0.13 & 0.14 & 0.15 & 0.16 & 0.17 & 0.18 & 0.19 \\
\hline 0.2 & 0.21 & 0.22 & 0.23 & 0.24 & 0.25 & 0.26 & 0.27 & 0.28 & 0.29 & 0.3 & 0.31 \\
\hline 0.32 & 0.33 & 0.34 & 0.35 & 0.365 & 0.385 & 0.405 & 0.425 & 0.445 & 0.465 & 0.485 & \\
\hline
\end{tabular}

$1997 / 03-2006 / 03$

\begin{tabular}{|l|l|l|l|l|l|l|l|l|l|l|l|}
\hline 0.03 & 0.04 & 0.05 & 0.06 & 0.07 & 0.08 & 0.09 & 0.1 & 0.11 & 0.12 & 0.13 & 0.14 \\
\hline 0.15 & 0.16 & 0.17 & 0.18 & 0.19 & 0.2 & 0.21 & 0.22 & 0.23 & 0.24 & 0.25 & 0.26 \\
\hline 0.27 & 0.28 & 0.29 & 0.3 & 0.31 & 0.32 & 0.33 & 0.34 & 0.35 & 0.36 & 0.37 & 0.38 \\
\hline 0.39 & 0.4 & & & & & & & & & & \\
\hline
\end{tabular}

2006/06 - 2019/11

\begin{tabular}{|l|l|l|l|l|l|l|l|l|l|l|l|}
\hline 0.02 & 0.0325 & 0.0375 & 0.0425 & 0.0475 & 0.0525 & 0.0575 & 0.0625 & 0.0675 & 0.0725 & 0.0775 & 0.0825 \\
\hline 0.0875 & 0.0925 & 0.1 & 0.11 & 0.12 & 0.13 & 0.14 & 0.15 & 0.16 & 0.17 & 0.18 & 0.19 \\
\hline 0.2 & 0.21 & 0.22 & 0.23 & 0.24 & 0.25 & 0.26 & 0.27 & 0.28 & 0.29 & 0.3 & 0.31 \\
\hline 0.32 & 0.33 & 0.34 & 0.35 & 0.365 & 0.385 & 0.405 & 0.425 & 0.445 & 0.465 & 0.485 & 0.32 \\
\hline
\end{tabular}

NOAA NCEI official archives show different frequencies bands for the same data.

$1990 / 10-1995 / 05==40$ frequencies

\begin{tabular}{|l|l|l|l|l|l|l|l|l|l|l|l|}
\hline 0.01 & 0.02 & 0.03 & 0.04 & 0.05 & 0.06 & 0.07 & 0.08 & 0.09 & 0.1 & 0.11 & 0.12 \\
\hline 0.13 & 0.14 & 0.15 & 0.16 & 0.17 & 0.18 & 0.19 & 0.2 & 0.21 & 0.22 & 0.23 & 0.24 \\
\hline 0.25 & 0.26 & 0.27 & 0.28 & 0.29 & 0.3 & 0.31 & 0.32 & 0.33 & 0.34 & 0.35 & 0.36 \\
\hline 0.37 & 0.38 & 0.39 & 0.4 & & & & & & & & \\
\hline
\end{tabular}

$1995 / 06-1997 / 02==47$ frequencies

\begin{tabular}{|l|l|l|l|l|l|l|l|l|l|l|l|}
\hline 0.02 & 0.0325 & 0.0375 & 0.0425 & 0.0475 & 0.0525 & 0.0575 & 0.0625 & 0.0675 & 0.0725 & 0.0775 & 0.0825 \\
\hline 0.0875 & 0.0925 & 0.1 & 0.11 & 0.12 & 0.13 & 0.14 & 0.15 & 0.16 & 0.17 & 0.18 & 0.19 \\
\hline 0.2 & 0.21 & 0.22 & 0.23 & 0.24 & 0.25 & 0.26 & 0.27 & 0.28 & 0.29 & 0.3 & 0.31 \\
\hline 0.32 & 0.33 & 0.34 & 0.35 & 0.365 & 0.385 & 0.405 & 0.425 & 0.445 & 0.465 & 0.485 & \\
\hline
\end{tabular}

$1997 / 03-1997 / 03==60$ frequencies

\begin{tabular}{|l|l|l|l|l|l|l|l|l|l|l|l|}
\hline 0.01 & 0.02 & 0.03 & 0.0325 & 0.0375 & 0.04 & 0.0425 & 0.0475 & 0.05 & 0.0525 & 0.0575 & 0.06 \\
\hline 0.0625 & 0.0675 & 0.07 & 0.0725 & 0.0775 & 0.08 & 0.0825 & 0.0875 & 0.09 & 0.0925 & 0.1 & 0.11 \\
\hline 0.12 & 0.13 & 0.14 & 0.15 & 0.16 & 0.17 & 0.18 & 0.19 & 0.2 & 0.21 & 0.22 & 0.23 \\
\hline 0.24 & 0.25 & 0.26 & 0.27 & 0.28 & 0.29 & 0.3 & 0.31 & 0.32 & 0.33 & 0.34 & 0.35 \\
\hline 0.36 & 0.365 & 0.37 & 0.38 & 0.385 & 0.39 & 0.4 & 0.405 & 0.425 & 0.445 & 0.465 & 0.485 \\
\hline
\end{tabular}

$1997 / 04-2006 / 05==40$ frequencies

\begin{tabular}{|l|l|l|l|l|l|l|l|l|l|l|l|}
\hline 0.01 & 0.02 & 0.03 & 0.04 & 0.05 & 0.06 & 0.07 & 0.08 & 0.09 & 0.1 & 0.11 & 0.12 \\
\hline 0.13 & 0.14 & 0.15 & 0.16 & 0.17 & 0.18 & 0.19 & 0.2 & 0.21 & 0.22 & 0.23 & 0.24 \\
\hline 0.25 & 0.26 & 0.27 & 0.28 & 0.29 & 0.3 & 0.31 & 0.32 & 0.33 & 0.34 & 0.35 & 0.36 \\
\hline 0.37 & 0.38 & 0.39 & 0.4 & & & & & & & & \\
\hline
\end{tabular}

$2006 / 06-2006 / 06==60$ frequencies

\begin{tabular}{|l|l|l|l|l|l|l|l|l|l|l|l|}
\hline 0.01 & 0.02 & 0.03 & 0.0325 & 0.0375 & 0.04 & 0.0425 & 0.0475 & 0.05 & 0.0525 & 0.0575 & 0.06 \\
\hline 0.0625 & 0.0675 & 0.07 & 0.0725 & 0.0775 & 0.08 & 0.0825 & 0.0875 & 0.09 & 0.0925 & 0.1 & 0.11 \\
\hline 0.12 & 0.13 & 0.14 & 0.15 & 0.16 & 0.17 & 0.18 & 0.19 & 0.2 & 0.21 & 0.22 & 0.23 \\
\hline 0.24 & 0.25 & 0.26 & 0.27 & 0.28 & 0.29 & 0.3 & 0.31 & 0.32 & 0.33 & 0.34 & 0.35 \\
\hline 0.36 & 0.365 & 0.37 & 0.38 & 0.385 & 0.39 & 0.4 & 0.405 & 0.425 & 0.445 & 0.465 & 0.485 \\
\hline
\end{tabular}

2006/07 $-2019 / 11==47$ frequencies

\begin{tabular}{|l|l|l|l|l|l|l|l|l|l|l|l|}
\hline 0.02 & 0.0325 & 0.0375 & 0.0425 & 0.0475 & 0.0525 & 0.0575 & 0.0625 & 0.0675 & 0.0725 & 0.0775 & 0.0825 \\
\hline 0.0875 & 0.0925 & 0.1 & 0.11 & 0.12 & 0.13 & 0.14 & 0.15 & 0.16 & 0.17 & 0.18 & 0.19 \\
\hline 0.2 & 0.21 & 0.22 & 0.23 & 0.24 & 0.25 & 0.26 & 0.27 & 0.28 & 0.29 & 0.3 & 0.31 \\
\hline 0.32 & 0.33 & 0.34 & 0.35 & 0.365 & 0.385 & 0.405 & 0.425 & 0.445 & 0.465 & 0.485 & \\
\hline
\end{tabular}

